\title{
A review on model updating of joint structure for dynamic analysis purpose
}

\author{
S.N. Zahari ${ }^{1}$, A.A.R. Zakaria ${ }^{1}$ M.S.M. Sani ${ }^{1, a}$ and I. Zaman ${ }^{2}$ \\ ${ }^{1}$ Advanced Structural Integrity and Vibration Research (ASIVR), Faculty of Mechanical Engineering, Universiti Malaysia Pahang, 26600 \\ Pekan, Pahang, Malaysia \\ ${ }^{2}$ Department of Engineering Mechanics, Faculty of Mechanical and Manufacturing Engineering, Universiti Tun Hussein Onn Malaysia \\ 86400 Parit Raja, Batu Pahat, Johor, Malaysia
}

\begin{abstract}
Structural joints provide connection between structural element (beam, plate etc.) in order to construct a whole assembled structure. There are many types of structural joints such as bolted joint, riveted joints and welded joints. The joints structures significantly contribute to structural stiffness and dynamic behaviour of structures hence the main objectives of this paper are to review on method of model updating on joints structure and to discuss the guidelines to perform model updating for dynamic analysis purpose. This review paper firstly will outline some of the existing finite element modelling works of joints structure. Experimental modal analysis is the next step to obtain modal parameters (natural frequency $\&$ mode shape) to validate and improve the discrepancy between results obtained from experimental and the simulation counterparts. Hence model updating will be carried out to minimize the differences between the two results. There are two methods of model updating; direct method and iterative method. Sensitivity analysis employed using SOL200 in NASTRAN by selecting the suitable updating parameters to avoid ill-conditioning problem. It is best to consider both geometrical and material properties in the updating procedure rather than choosing only a number of geometrical properties alone. Iterative method was chosen as the best model updating procedure because the physical meaning of updated parameters are guaranteed although this method required computational effort compare to direct method.
\end{abstract}

\section{Introduction}

Joints are essential parts of complex structure and play a vital role in the assembled structure's behaviors (e.g. flexibility and damping). There are various kinds of joints used in the engineering structure, such as welded joints, bolted joints and riveted joints. These connecting elements contribute significantly to dynamic behaviors of a complete structure. Assuming rigid connections between substructures in modelling without considering the joints effects may lead to different characteristics compared to actual physical structure.

Dynamic properties of a joint structure can be investigated via two methods which by numerical and experimental analysis. The most commonly used numerical method is finite element (FE) method which is used to simulate the behavior of real systems and several models have been presented in this literature. The purpose of numerical modelling can be classified into three main categories which are analysis, prediction and design. Normally, the numerical model for FE analysis of the associated actual engineering structure is constructed on the basis of highly idealized engineering design that may not fully represent all the physical and geometrical aspects of the actually built structure analysis. Therefore,
FE predictions are often called into question when they are in conflicts with experimental result. Based on previous studies $[1,2]$, the discrepancy between numerical and experimental results may exceed $10 \%$ or sometime even $40 \%$ error. Incorrect modelling of boundary condition, modelling of joints and difficulties modelling with damping that led to existing of difference between numerical and test result.

This has led to the development of model updating technique also known as model calibration or in simplified terms, parameter estimation or identification. The main purpose of model updating is to reduce the errors between numerical and test results. Model updating often implemented by analyzing the degree to which a finite element model represent a single set of experimental data $[3,4]$. There are two methods of model updating which are direct method and sensitivity method. In model updating, the selection of updating parameter is the most important task. If numerical predictions are insensitive to a chosen parameter, then updating will result in a change to the parameter of uncertain value, because the difference between predictions and results has been reconciled by changes to other (more sensitive) parameters that might be less in need of updating. The result, in that case will be an updated model which

${ }^{\mathrm{a}}$ Corresponding author: mshahrir@ump.edu.my 
replicates the measurements but lacks physical meaning. The updated parameters should be justified physically and the quality of the final model should be assessed within the operating range. There are three aspects that lead to a credibility for updated structural; robustness to uncertainties, fidelity to data and confidence in predictions $[5,6]$.

This review paper first outlines on FE modelling for different kinds of joints structure from previous studies with attention given to spot welded modelling joints due to its continuous relevance to this review paper on model updating. The identification of updating parameters and stochastic model updating will be emphasized in the second section. The following section will provide a brief explanation on the method of model updating using sensitivity approach.

\section{FE modelling of joints structure}

Many engineering structures were assembled from components by using variety of connections such as bolted, riveted, fastener and welded joints. For a simple structure, modelling and numerical predictions have been developed for decades. However prediction of dynamic characteristic for structure with joints is not an easy task due to its complexities in modelling the joints. Joints add damping to the structural system and decrease overall stiffness thus changing the overall dynamics characteristic of the system. Due to this effect, modelling of the joint has become more and more significant.

Bolted joints are widely used in joining the component especially in modern aircraft application due to its characteristics; easily disassembled, maintained and inspected. Many researches on joints have been conducted by scientists and engineers since early 1970 [7-9]. On top of that, they have tried to understand the characteristics of joints and simulate their findings into analytical modelling. Gaul and Nitsche [7] concentrated on describing nonlinear transfer behaviour of frictional interface using constitutive and phenomenological models. Constitutive models are based on interface physics by describing the friction phenomena in local manner while phenomenological model depends on experimental observation. The paper covered on Jenkinelement models (also known as an elasto-slip model) that consisting of linear springs and Coulomb friction element were used to represent the friction interface of the bolted joints. It is shown that this model has a potential to simulate friction force in a bolted joint very well. Iranzad [10] also utilized a constitutive model with a thin layer of elasto-plastic material to model nonlinear behaviour of bolted joints. The thin layer elastic material properties represent the joint linear behaviour at low vibration levels. Yoo et al. [8] proposed cone frusta method for jointed part and spring elements to represent the contact effects occurring in the interface area in simulating the dynamic behaviour of bolted joints structure with a large interface structure. Another bolted joint model presented by Wang et al. [9] introduced the strain rate dependant elastic modulus into the spring mass model. On the other hand, Rutman et al. [11] identified the modelling techniques of the bolted joint used to connect with different types of components in NASTRAN such as spring elements (CELAS and CBUSH), connector elements (CBAR and CBEAM) and rigid connection element (RBE2). The combination of element used to idealise the bending and the shear of the fastener shank. It also bring elastic bearing stiffness of the plate and fasteners at the contact surface. These proposed models are capable to predict the strain rate dependant stiffness and strength of composite bolted joint under static and dynamic loading.

Compared to bolted and riveted joints, welded joints are permanent and reliable evaluation of the behaviour of the welds is always a concern. There are many local effects such as geometrical irregularities, residual stresses, materials inhomogeneity and defects during welding process that are not to be considered by FE modelling lead to difficulties to model the welded joints. In real structure, there are thousands of spot welds and modelling the detailed model will be a challenge. A very detailed model produces a detailed and smooth stress field, but it may provide less accurate prediction of stiffness for real spot welds and their effect on the rest of the structure. However for vibration analysis purposes, only simple models that represent the stiffness characteristic needed to predict their influence on the rest of the structure. Several previous research [12-15] focused on modelling of spot weld been discussed by [16] for stress analysis and stiffness simulation. For latest vibration analysis and model updating purposes, the spot weld modelling being carried out by some of these researchers [17-19].

Abu Husain et al. [17] developed FE model of spot welds using CWELD element for dynamic prediction. CWELD element represented by two nodes special shear flexible beam type element with 12 DOFs (six for each node) and all nodes are connected to their corresponding patches with constraints from the Kirchhoff shell theory [20]. This type of model can be used to represent laser spot weld with good accuracy by selecting the right updating parameters. When this CWELD element modelling to be used in dynamic analysis of structure of similar constructions, it is recommended to put the value for Young modulus of the patch three times the value for Young modulus of weld dynamic analysis of structure. Another spot weld model was proposed by Kuratani [18]. The model known as Area Contact Model 2 (ACM2) using multi point constraint (MPC) that designed in ANSYS software that having a same features as in NASTRAN. The ACM2 model consisted of a single solid element connecting the upper and lower shell element with RBE3 (rigid connection element). The RBE3 element is an interpolation element and automatically generates internal MPC equations in finite element analysis process. These models can be employed to investigate the effects of mesh size on modal properties (natural frequency and mode shape). ACM2 model is relatively sensitive to mesh size in patch area. When the size of mesh in patch area increases, the natural frequency also increases. This leads to the increasing stiffness at the centre of the patch due to the patch area size increase. The higher and lower mesh size will cause 
the loss of stiffness. Therefore, patch area must be meshed with care. From this study, it concludes that the proper shell element size in the patch area is dependent on the solid element size determined from the diameter of a weld nugget. The recommended range of the ratio of element sizes of the shell to the solid elements is between 1.0 and 1.5. On the other hand, Alvarez [19] also proposed new model of spot weld that imposes a surface to surface connection between two structures using simple spring element and multipoint constraint connection (MPC) therefore the coincident meshes are not required. Area of the spot weld is consistent to spot weld dimension whereas rotational stiffness is proportional to the spot weld radius. This model develops a connection model by adding an equivalent rotational stiffness to the system using an array of translational spring instead of adding stiffness to rotational degree of freedom (DOF). This type of model built to avoid the problem of sensitivity to element size that leads to poor convergence. When the proposed spot model had been used, the average sensitivity of element size is reduced almost five times from the actual value.

\section{Experimental modal analysis}

Experimental modal analysis or modal testing has grown steadily in popularity for the past several decades. Modal testing is defined as the study of dynamics characteristic of a mechanical structure. The setup and the instrumentation used influence the experimental result in modal testing [21]; therefore to obtain the accurate result, modal testing should be carried out in a free-free boundary condition. EMA is used to extract modal parameters (natural frequency, mode shape, damping ratio, modal vectors and modal scaling). The structure will be excited by certain inputs (hammer, shaker) and sensing mechanism will be used to measure the input force in order to produce a set of frequency response function (FRF's) that contain inherent dynamic properties of a structure. EMA plays an important part in design and analysis of structures and conducted to validate the results from simulation models before they can be used for further detailed analysis. Finite element model updating will be performed to reduce the discrepancy between modal testing and numerical results.

\section{Identification of updating parameters}

The choice of parameters is a critical part in model updating and to produce well-conditioned updating problem, it is necessary to select those updating parameters which will be most effective in producing a genuine improvement in modelling the structure [3]. Parameters that have been selected will be adjusted to minimize a penalty function based on residual between an experimental set and the corresponding numerical method. Normally the parameter that sensitive to the data should be taken into consideration as updating parameters but this is cannot be the main reason for selecting updating parameters. Updating parameters should be chosen with the aim of correcting recognized uncertainties in the model and the data should be sensitive to them.

There are two main groups of uncertainties in analytical modelling; physical uncertainties and numerical uncertainties. Boundary and initial condition, material properties, geometry and load are classified as physical uncertainties meanwhile for conceptual and mathematical modelling, discretization error, numerical solution and human mistakes known as numerical uncertainties [4]. Once the uncertainties are being considered, a deterministic problem will then change to non-deterministic problem (stochastic). It is very useful and highly recommended to explore numerical predictions on behavior of structure with uncertainties and many previous researches studied on stochastic model updating approach using two methods [5-15]; Monte Carlo simulation and perturbation method. For this paper, it focuses on spot welded structure and as simplified in the first section, the joints structure could be considered less accurately modelled due to the uncertainties in the structural parameters such as modulus elasticity and diameter of the weld, mass density, boundary condition etc. Therefore, the parameterization of the inaccurate parts of the welded structure is important.

The material parameters, thickness and cross sectional dimensional tends to be most powerful updating parameters because they often apply throughout a FE mesh affecting a large number of elements therefore a small change in these parameters will affect the natural frequency very considerably [16]. There is a study by Abu Husain et al [12] that shows that selecting some of material properties as the updating parameters provide better convergence than those updated by using only the thickness parameter. From these findings, it stated that in numerical predictions it is best to consider both geometrical and material properties in the updating procedure rather than choose only a number of geometrical properties alone.

Then, Abu Husain et al. also presented in the next research [13] on welded structure as the main uncertainties employed a perturbation method that being used by Haddad Khodaparast et al. [11] to investigate variability that exist between a set of nominally identical structures. The perturbation methods are used for estimation of means and covariance of updating parameters and two approaches of parameter weighting matrix assignment are explained; one approach using three parameters from the welds and the other used eight parameters which are five from the component itself and three are from the weld. The latter approach is in a very good agreement with experimental data and excellent correlation between predicted and measured covariance of the output is achieved compare to the first approach that only demonstrates good correlation between the predicted mean natural frequencies and their measured data, but poor correlation is obtained between the predicted and measured covariance of the outputs [13].

\section{Model Updating Procedure}


The FE method is a well-known technique that is used to analyze the behavior of a structure subjected to a variety of loads. The computation of undamped natural frequency and mode shapes is usually conducted using normal mode analysis (SOL103) in NASTRAN [17]. The result from analysis is then compared with experimental counterparts for validation purposes. The correlation between the results from these two methods for natural frequencies obtained directly but for mode shapes, there should be further analysis to validate mode shapes. Modal assurance criterion (MAC) values range from 0 and 1 can be used to predict the correlation and to pair the mode shapes vectors from experimental and simulation result. The comparisons between the numerical modal properties (i.e., the natural frequencies and mode shapes) and their measured counterparts will normally reveal some discrepancies between the two approaches, thus the FE model updating method [3] should be performed to minimise the errors. Model updating can be categorized into two different classes: direct (non-iterative) method and sensitivity (iterative) method. Direct method is known as representational method because their ability in replicating measured data [3]. But the measurement and numerical data will show some discrepancies due to existing error such as noise and model inadequacies. Using direct method, if the updated model exactly reproduces inaccurate measurement, any subsequent analysis will be inaccurate. Although these methods are computationally cheaper and reproduce the measured model data exactly, they violate structural connectivity and updated structural matrices are difficult to interpret.

Iterative method also known as sensitivity method provides wide choices of updating parameters, structural connectivity can be easily maintained and corrections suggested in the selected parameters can be physically interpreted. Iterative methods are based on minimizing an objective function that is generally a nonlinear function of selected updating parameters. Iterative method used either eigendata or frequency response function (FRF) to construct objective function. For analytical model updating, Collins [18] focused on the eigendata sensitivity meanwhile Lin and Ewins [19] used FRF data. Modak et al. [20] and his next research [21] neglecting the damping, only focusing on comparison of response function method (RFM) and inverse eigensensitivity method with an objective to study the accuracy with which they predicted the corrections required in an FE model. One of sensitivity method is the Design Sensitivity and Optimization Code (SOL 200) in Nastran [22] is used for updating, and an objective function is based on residuals between measurement data (natural frequency, mode shapes, FRF, etc.) and their predictions are set for minimisation procedure. The objective functions based on eigenvalues [3] is defined as Eq. (1):

$$
J=\sum_{i=1}^{n} W_{i}\left(\frac{\lambda_{i}^{f e}}{\lambda_{i}^{e x p}}-1\right)^{2}
$$

Where:

$J$ is the objective function,

$W_{i}$ is the weighting coefficient,

$\lambda_{i}^{f e}$ is the $i^{\text {th }}$ predicted eigenvalue from finite element analysis, $\lambda_{i}^{e x p}$ is the $i^{\text {th }}$ experimental eigenvalue, $n$ is the machining time in minutes.

The procedure will be repeated until the convergence has been accomplished where the difference between objective function $(J)$ value from consecutively iteration become smaller. SOL 200 in NASTRAN is employed for model updating through procedures [23] illustrate in Figure 1. The optimisation algorithm uses partial derivatives of a function to assist in a numerical search for optimisation procedure. The optimisation algorithms in NASTRAN generally belongs to the gradient based methods [24].

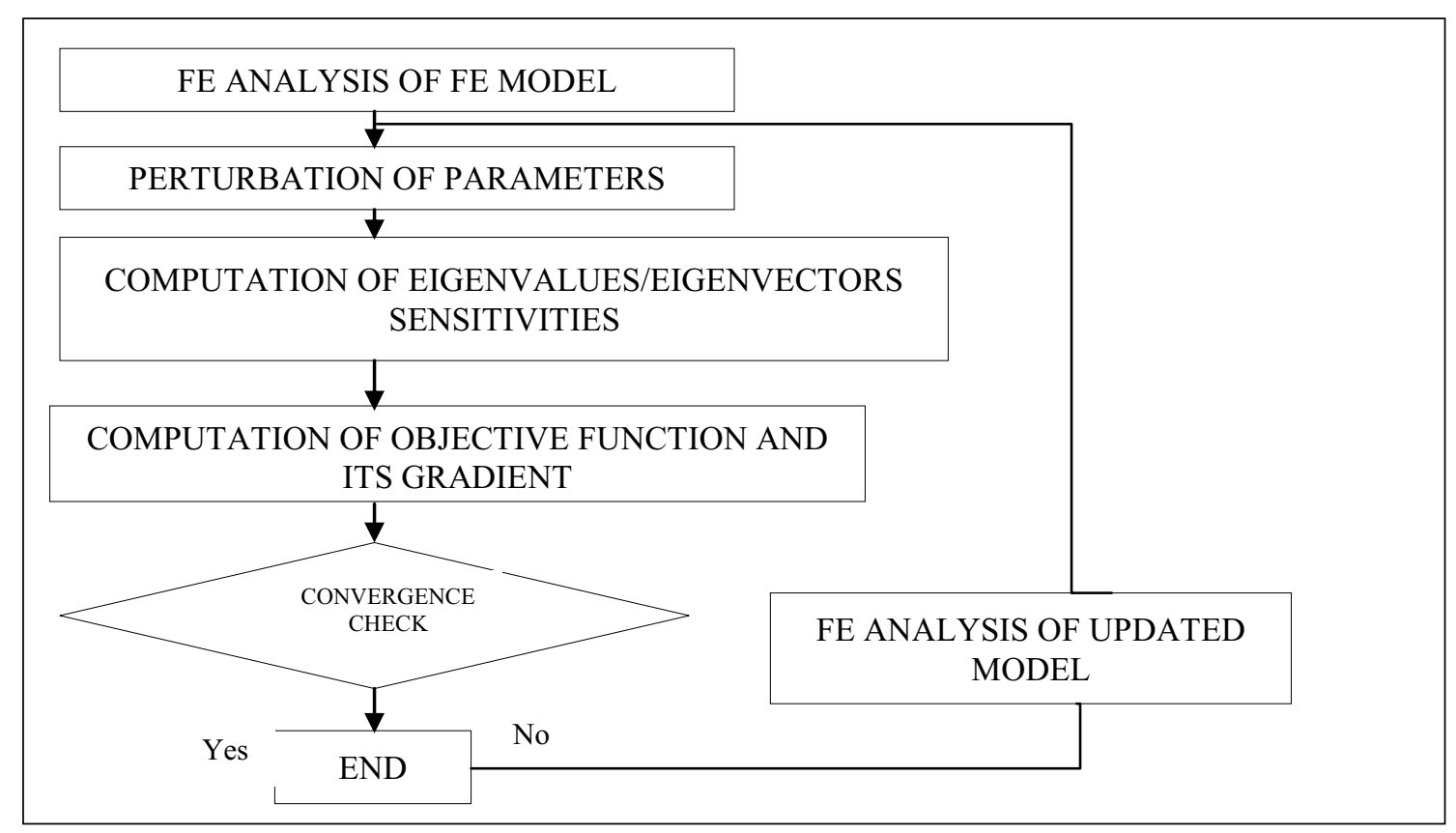

Figure 1. Procedure of model updating using SOL200 in NASTRAN 


\section{Conclusion}

In this paper, a simple review of model updating on the joint structure has been presented. Currently, FE modelling for joints regarded as an important part in model updating. Without accurate modelling, model updating cannot be carried out significantly. The selection of updating parameters also plays a crucial part in performing model updating. It is best to consider both geometrical and material properties in the updating procedure rather than choosing only a number of geometrical properties alone. SOL200 in NASTRAN software recommended as the one of iterative methods for model updating procedure by minimizing objective function in order to decrease the difference between results obtained through predicted model parameters and experimental counterparts. This is due to their iterative nature that provides guarantee for physical meaning of updated parameters even though this method required computational effort.

\section{Acknowledgements}

The author would like to greatly acknowledge the support by focus group of Advanced Structural Integrity of Vibration Research (ASIVR) and Universiti Malaysia for providing all the equipment used for this project and special thank for Knowledge Transfer Programme (KTP) and Ministry Of Education for financial assistance support.

\section{References}

1. Brownjohn, J.M. and P.-Q. Xia, Dynamic assessment of curved cable-stayed bridge by model updating. Journal of Structural Engineering, (2000). 126(2): p. 252-260.

2. Živanović, S., A. Pavic, and P. Reynolds, Finite element modelling and updating of a lively footbridge: The complete process. Journal of Sound and Vibration, (2007). 301(1-2): p. 126-145.

3. Friswell, M. and J.E. Mottershead, Finite element model updating in structural dynamics. Vol. 38. (1995): Springer Science \& Business Media.

4. Dascotte, E. The use of FE model updating and probabilistic analysis for dealing with uncertainty in structural dynamics simulation. in Japan Modal Analysis Conference, Sept. (2003).

5. Schuëller, G.I., et al., Uncertainty Analysis of a Large-Scale Satellite Finite Element Model. Journal of Spacecraft and Rockets, (2009). 46(1): p. 191-202.

6. Mares, C., J. Mottershead, and M. Friswell, Stochastic model updating: part 1-theory and simulated example. Mechanical Systems and Signal Processing, (2006). 20(7): p. 1674-1695.

7. Mottershead, J., et al., Stochastic model updating: part 2-application to a set of physical structures. Mechanical Systems and Signal Processing, (2006). 20(8): p. 2171-2185.
8. Fang, S.-E., W.-X. Ren, and R. Perera, A stochastic model updating method for parameter variability quantification based on response surface models and Monte Carlo simulation. Mechanical Systems and Signal Processing, (2012). 33(0): p. 83-96.

9. Rui, Q., H. Ouyang, and H.Y. Wang, An efficient statistically equivalent reduced method on stochastic model updating. Applied Mathematical Modelling, (2013). 37(8): p. 6079-6096.

10. Bao, N. and C. Wang, A Monte Carlo simulation based inverse propagation method for stochastic model updating. Mechanical Systems and Signal Processing, (2015).

11. Khodaparast, H.H., J.E. Mottershead, and M.I. Friswell, Perturbation methods for the estimation of parameter variability in stochastic model updating. Mechanical Systems and Signal Processing, (2008). 22(8): p. 1751-1773.

12. Husain, N.A., H.H. Khodaparast, and H. Ouyang. Parameter selections for stochastic uncertainty in dynamic models of simple and complicated structures. in Proceedings of the 10th International Conference on Recent Advances in Structural Dynamics, University of Southampton, Southampton. (2010).

13. Husain, N.A., et al. Application of the Perturbation Method With Parameter Weighting Matrix Assignments for Estimating Variability in a Set of Nominally Identical Welded Structures. in ASME 2010 10th Biennial Conference on Engineering Systems Design and Analysis. (2010). American Society of Mechanical Engineers.

14. Govers, Y. and M. Link, Stochastic model updating-Covariance matrix adjustment from uncertain experimental modal data. Mechanical Systems and Signal Processing, (2010). 24(3): p. 696-706.

15. Chen, H.-P. and T.S. Maung, Regularised finite element model updating using measured incomplete modal data. Journal of Sound and Vibration, (2014). 333(21): p. 5566-5582.

16. Mottershead, J.E., M. Link, and M.I. Friswell, The sensitivity method in finite element model updating: $a$ tutorial. Mechanical Systems and Signal Processing, (2011). 25(7): p. 2275-2296.

17. Sitton, G., MSC/NASTRAN basic dynamic analysis user's guide. Macneal-Schwendler Co, (1997).

18. Collins, J.D., et al., Statistical Identification of Structures. AIAA Journal, (1974). 12(2): p. 185-190.

19. Lin, R.M. and D.J. Ewins, Analytical model improvement using frequency response functions. Mechanical Systems and Signal Processing, (1994). 8(4): p. 437-458.

20. Modak, S.V., T.K. Kundra, and B.C. Nakra, Comparative study of model updating methods using simulated experimental data. Computers \& Structures, (2002). 80(5-6): p. 437-447.

21. Modak, S.V., T.K. Kundra, and B.C. Nakra, Model updating using constrained optimization. Mechanics Research Communications, (2000). 27(5): p. 543551.

${ }^{a}$ Corresponding author: mshahrir@ump.edu.my 
22. Husain, N.A., et al., Finite-element modelling and updating of laser spot weld joints in a top-hat structure for dynamic analysis. Proceedings of the Institution of Mechanical Engineers, Part C: Journal of Mechanical Engineering Science, (2010). 224(4): p. 851-861.

23. Moore, G.J., MSC/NASTRAN design sensitivity and optimization: user's guide, version 68. (1994): MacNeal-Schwendler Corporation.

24. Mares, C., M. Friswell, and J. Mottershead, Model updating using robust estimation. Mechanical Systems and Signal Processing, (2002). 16(1): p. 169-183. 